\title{
Utilização do custo-meta na formação de preços em entidades prestadoras de serviços educacionais.
}

\begin{abstract}
Alzenir José de Vargas
Mestrado em Ciências Contábeis pela Universidade Regional de Blumenau Rua Antônio da Veiga, 140. Sala D 201. Bairro Victor Konder Blumenau/SC. CEP: 89012-900

E-mail: ajvargas@al.furb.br

Jorge Eduardo Scarpin Doutorado em Controladoria e Contabilidade pela FEA/USP Professor da Universidade Federal do Paraná - UFPR Av. Prefeito Lothário Meissner, 632. 1ํ. Andar. Campus III. Jd. Botânico. Curitiba/PR. CEP: $80210-170$ E-mail: jscarpin@gmail.com
\end{abstract}

\section{RESUMO}

A implantação de ferramentas estratégicas no processo de gestão das Instituições de Ensino Superior Privado vem passando por alterações significativas nos últimos anos, visto a expansão da oferta de vagas e a redução no número de alunos. A reestruturação dos sistemas de custos é considerada estratégica no atendimento das expectativas por parte dos acadêmicos em relação aos serviços oferecidos e aos preços cobrados. O presente artigo tem como objetivo analisar a importância, na percepção dos acadêmicos, dos principais fatores geradores de custos que compõem o valor da mensalidade. A coleta de dados foi realizada com a aplicação de um questionário estruturado, com uma amostra de cento e setenta e seis acadêmicos de três cursos de graduação de uma instituição de ensino superior. Utilizou-se a análise descritiva identificando o resultado individual por curso e a média obtida entre eles. As descobertas destacam a importância significativa relacionada aos professores, estrutura de biblioteca, laboratórios, atendimento online e a realização de atividades complementares oferecidas pela instituição. As atividades consideradas pouco importantes estão relacionadas aos serviços complementares de livraria e restaurante universitário. O estudo possibilitou identificar os principais fatores considerados importantes na composição do custo repassado no valor da mensalidade.

Palavras-chave: Custo Meta. Instituição de Ensino Superior Privado. Valor. 


\section{ABSTRACT}

The implementation of strategic tools in the process of management of private higher education institutions has been going through significant changes in recent years, since the expansion of supply openings and the reduction in the number of students. The reorganization of costs is considered strategic in attendance expectations on the part of scholars for the services provided and the prices charged. This article aims to identify and analyze the importance, in the perception of the scholars, the main cost factors generators that make up the value of the monthly fee. The data collection was carried out by applying a structured questionnaire, with a sample of one hundred and seventysix academics of three undergraduate courses of a higher education institution. It was used descriptive analysis to identify the individual result obtained in the course and the average between them. The findings highlight the importance related to teachers, library structure, laboratories, online service and complementary activities offered by the institution. The activities considered unimportant are related to the complementary services of university bookstore and restaurant. The study made it possible to identify the main factors considered important in the composition of cost passed on the value of the monthly fee.

Keywords: Target Costing. Private Higher Education Institution. Value.

\section{INTRODUÇÃO}

A abertura de novas possibilidades de criação de entidades prestadoras de serviços educacionais vem apresentando necessidades de adequações nos procedimentos relacionados às políticas de valoração dos serviços educacionais prestados. A pulverização de entidades educacionais com e sem fins lucrativos está exigindo que os gestores das entidades sem fins lucrativos adotem novas medidas e estratégias de manutenção de suas atividades. Com características bem específicas, estas entidades só se mantêm com o pagamento de mensalidades dos seus alunos.

Giollo (2006, p. 68) destaca que estas instituições se denominam "universidades particulares não-confessionais, onde existe uma vinculação e um compromisso direto com a comunidade, de oferecer um ensino de qualidade e com baixo custo." Porém, a entidade precisa gerir os recursos arrecadados com os pagamentos dos alunos a fim de atingir os objetivos que propõe, cobrindo seus custos e fazendo novos investimentos. 
O aumento das vagas oferecidas no ensino superior faz com que as entidades busquem novas alternativas de manutenção e expansão de suas atividades. A opção por uma nova configuração no processo de gestão obriga o gestor a buscar alternativas para a fidelização do aluno. A questão econômica relacionada ao valor cobrado, muitas vezes, é considerada como fator decisivo para o aluno. Neste sentido, a adequação dos preços passa a ser uma questão estratégica para estas instituições.

Knuth (2005) destaca que o "preço" está relacionado diretamente com o poder de compra dos consumidores, e um dos fatores que influenciam a sobrevivência das empresas no mercado é o custo. No entanto, os preços dos produtos e serviços normalmente são estabelecidos pelo mercado, influenciando significativamente no resultado das entidades.

Horngren et al. (1997) destacam que as decisões de preços influenciam diretamente nas receitas das empresas. Estas receitas deverão ser superiores aos custos, mas a escolha por preços praticados pelo mercado faz com que os custos sejam adequados ao preço que o mercado está disposto a pagar pelo produto ou serviço. As limitações da entidade, em determinar o preço a ser cobrado pelo serviço prestado ou produto vendido, necessitam da adequação dos custos no projeto, passando de um planejamento de custos para um planejamento de resultados.

Assim, o objetivo do estudo é analisar a importância, na percepção dos alunos, dos principais fatores geradores de custos que compõem o valor da mensalidade em uma instituição de ensino superior privada sem fins lucrativos estabelecida no estado do Rio Grande do Sul. A adoção do custo-meta (targent consting) se apresenta como uma alternativa na formação do valor das mensalidades dos alunos, permitindo à administração da instituição identificar o que efetivamente o aluno considera importante no valor pago em sua mensalidade. Para a instituição de ensino é uma possibilidade interessante, visto que as decisões em relação ao valor das mensalidades podem ser localizadas em partes específicas da composição do custo.

Sakurai (1997) define que o "custo-meta é um processo estratégico de gerenciamento de custos para reduzir custos totais, nos estágios de planejamento e de 
desenho do produto." A definição do custo-meta concentra os esforços integrados de todos os departamentos da empresa, possibilitando a redução dos custos aplicados na produção e incentivo à inovação.

$\mathrm{O}$ artigo é dividido em três partes, além da introdução e considerações finais. A primeira parte está relacionada à revisão de literatura sobre o assunto; a segunda parte trata da metodologia aplicada ao estudo; e a terceira e última apresenta a análise dos resultados do estudo aplicado.

\section{CUSTO - META (TARGET COSTING)}

Scarpin e Rocha (2000) relatam que o conceito de custo-meta vem da determinação dada pelos japoneses, genka kikaku, que significa "planejamento de lucros". O termo em inglês target costing, representa a tradução de custo-meta. Nos países de origem espanhola e francesa as expressões usadas são méthod du côut, objectif e costo objectivo, que significam custeio-objetivo.

Ansari et al. (1997, p. 11) definem custo-alvo (custo-meta) como sendo: "[...] um sistema de planejamento de lucros e gerenciamento de custos que é conduzido pelo preço, focado no cliente, centrado no projeto, que envolve diversas áreas da empresa."

Ainda em relação ao conceito de custo-alvo, (Rocha, 1999, p. 126) acrescenta:

Custo-alvo é o montante de custos que deve ser eliminado, ou aumentado, para que o custo estimado de um produto, ou serviço, se ajuste ao permitido, tendo em vista o custo de uso e de propriedade para o consumidor, o preço alvo e as margens objetivadas para cada elo da cadeia.

Sakurai (1997) ressalta como objetivo principal do custo-meta a redução de custos e a manutenção da alta qualidade do produto ou serviço, bem como um planejamento estratégico de lucros. A redução de custos está relacionada com os custos totais do produto ou serviço incluindo produção, marketing e usuários. Quando 
usado como planejamento estratégico de lucros, é parte integrante das informações do marketing, engenharia e produção.

Atkinson et al. (2008) afirmam que o custo-meta é o preço que os clientes estão dispostos a pagar por um produto menos o lucro desejado, ou seja, o custo que deve ser produzido por um produto, para atingir a meta de rentabilidade. Wernke (2008) corrobora com o referido conceito quando enfatiza que através do valor do custo de um produto a ser alcançado é possível cobrar o preço de venda suficiente para ser aceito pelo mercado consumidor e também determinar o lucro para os acionistas.

Wernke (2008) destaca que o processo de custeio pode ser dividido em três etapas: definir um custo-alvo viável do produto; disciplinar o processo de custeio-alvo a fim de garantir que seja atingido sempre que o produto for viável; e adequar o custo do produto à meta fixada, sem comprometer a funcionalidade e a qualidade do produto ou serviço, através da utilização da engenharia de valor e outras técnicas de redução de custo.

Horngren et al. (1997) observam quatro etapas a serem seguidas na implementação dos custeio-alvo com o objetivo de alcançar o preço meta. A primeira etapa consiste no desenvolvimento de um produto que atenda às necessidades dos potenciais consumidores; a etapa seguinte se refere à escolha do preço-meta, com base no valor aceito pelo consumidor e os preços dos concorrentes; a próxima etapa segue o cálculo do custo-meta unitário, subtraindo a meta do lucro operacional-meta unitário do preço-meta; e finalmente a realização de uma engenharia de valor para alcançar o custo-meta.

Cruz e Rocha (2008) descrevem seis princípios ou ideias fundamentais inerentes ao custeio-meta, onde:

- O custo é conduzido pelo preço, isto é, o preço de mercado define o custo do produto ou serviço;

- O foco está no cliente, portanto, consideram-se as exigências de mercado;

- O foco está no projeto, pois nesta etapa a maior parcela dos custos é definida; 
Utilização do custo-meta na formação de preços em entidades prestadoras de serviços educacionais. Alzenir José de Vargas, Jorge Eduardo Scarpin

- Deve haver um envolvimento das diversas áreas da empresa como controladoria, marketing, engenharia da produção, compras entre outras;

- A orientação está para o ciclo de vida do produto, tanto do produtor quanto do ponto de vista do consumidor, visando à otimização do custo total do consumidor;

- Devem considerar a cadeia de valor envolvendo fornecedores, distribuidores, prestadores de serviços, varejistas, etc.

Os sistemas de gerenciamento e controle de custos adotados em diferentes etapas do processo são apresentados no quadro a seguir, ressaltando que o custo padrão também é uma forma eficaz de planejar e controlar custos em determinada fase específica do processo (MARTINS, 2006: 315).

\begin{tabular}{|c|c|}
\hline Custo-meta & Custo-Padrão \\
\hline É definido no projeto & É usado no estágio da produção \\
\hline É um instrumento de planejamento de custos & É um instrumento de controle de custos. \\
\hline Está voltado para o mercado & Está voltado para a produção e para tecnologia. \\
\hline Faz parte do planejamento estratégico do lucro. & É um instrumento de controle de custo operacional. \\
\hline É uma técnica orientada pela engenharia & $\begin{array}{c}\text { É um instrumento de gerenciamento para } \\
\text { direcionar e focalizar o processo decisório fora da } \\
\text { engenharia de produção. }\end{array}$ \\
\hline $\begin{array}{l}\text { Depende da colaboração entre departamentos } \\
\text { onde necessita desta colaboração }\end{array}$ & $\begin{array}{l}\text { Está voltado para o departamento de contabilidade } \\
\text { que atua como coordenador e fornecedor de } \\
\text { informações. }\end{array}$ \\
\hline $\begin{array}{c}\text { É melhor na produção de grande variedade e } \\
\text { baixo volume. }\end{array}$ & $\begin{array}{c}\text { É eficaz quando aplicado na produção em massa } \\
\text { padronizada }\end{array}$ \\
\hline
\end{tabular}

Quadro 1: Comparação do Custo-Meta e o Custo Padrão Fonte: Adaptado de Sakurai (1997).

Os objetivos do custo-meta permitem uma análise desde a fase inicial do projeto até a mensuração final da margem gerada pelo produto ou serviço, destacados por Cruz e Rocha (2008) como sendo:

- Garantir a margem objetivada pela organização;

- A mudança de visão na formação do preço de venda;

- O custo determina o preço de venda; 
- Possibilita a realização de uma análise de custos considerando todo o ciclo de vida do produto, visando à otimização do custo total para o consumidor;

- A otimização do custo do produto não deve comprometer a qualidade do produto. Sakurai (1997) salienta que o principal objetivo do custo-meta é reduzir os custos totais, mantendo alta qualidade e também um planejamento de lucros. Cruz e Rocha (2008), considerando que as condições de preços impostas pelo mercado fazem com que as empresas busquem alternativas de adequação e gerenciamento de seus custos, encontram quatro razões da existência e delineamento do custo-alvo: o lucro é a garantia de sobrevivência da empresa; o custo conduzido pelo preço; a satisfação dos clientes; o custo é definido essencialmente no projeto.

Acerca dos conceitos e sistemática operacional do custo-meta, a Figura 1 traz uma síntese onde estabelece o custo máximo admissível, considerando todos os aspectos relacionados ao preço pago pelo consumidor, a identificação de características e satisfação dos clientes e estabelecendo o preço e a margem-alvo, tendo como base o custo máximo admissível para o produto ou serviço.

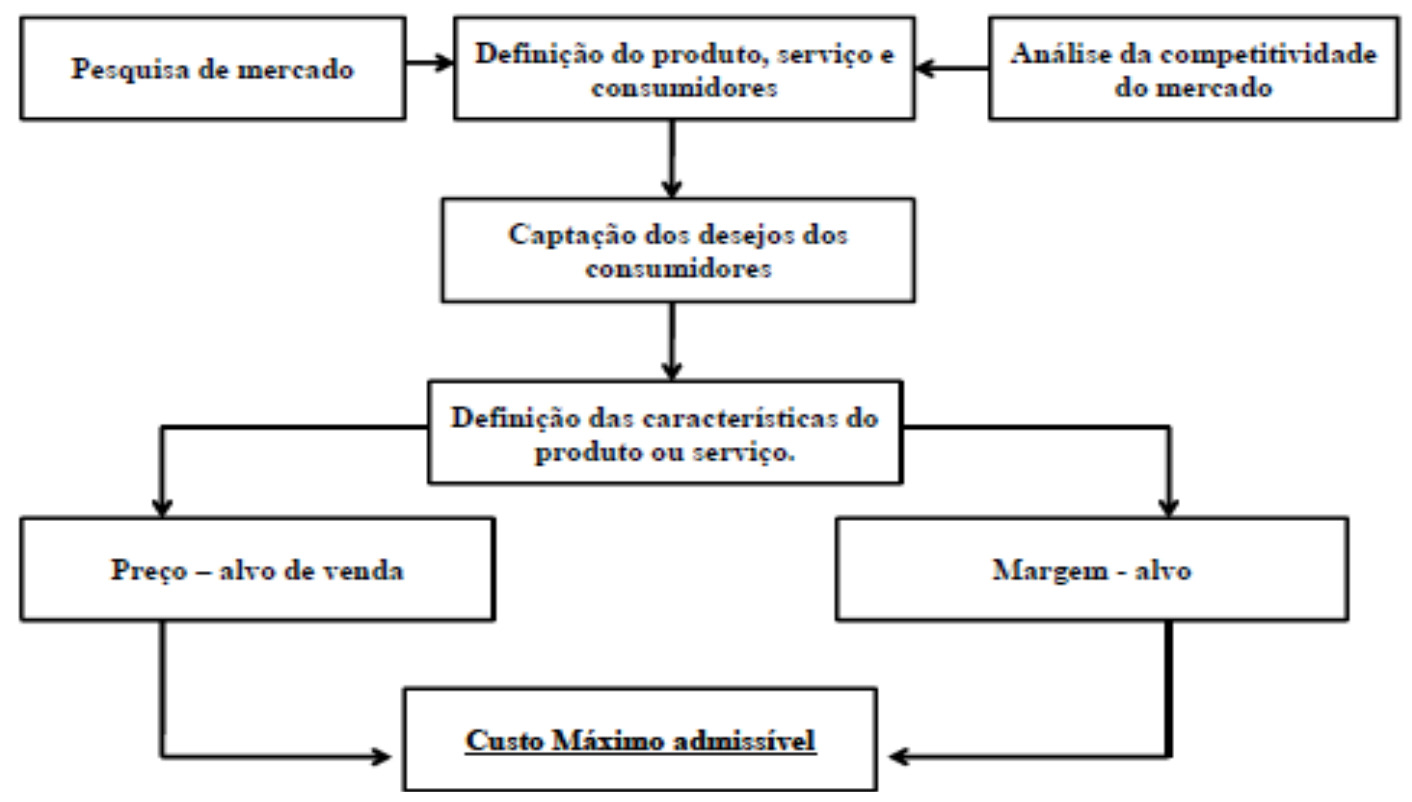

Figura 1: Custo Máximo Admissível Fonte: Adaptado de Hansen (2002). 
Normalmente, quando é abordado, o termo custo se relaciona diretamente com a produção de produtos, porém na prestação de serviços também apresenta sua estrutura de custos, pois segundo Chiavenatto (1990), serviços é o resultado de uma atividade produtiva.

Chimendes e Zaccaro (2008) reforçam que a unidade produtiva está diretamente relacionada ao consumidor, porque o contato é direto, não apresenta estoques de serviços e com isso seu desempenho financeiro está intimamente ligado à qualidade.

No quadro a seguir mostra-se um paralelo entre a abordagem tradicional de custo em relação ao custo-meta e com o objetivo de fixação dos conceitos desenvolvidos por Cruz e Rocha (2008), Atkinson et al. (2008), Sakurai (1997) e Wernke (2008).

\begin{tabular}{|c|c|}
\hline Custo-Meta & Custo Tradicional \\
\hline Preço determina o custo. & Custo determina o preço. \\
\hline $\begin{array}{c}\text { Planejamento de custos conduzido pela visão } \\
\text { de mercado. }\end{array}$ & $\begin{array}{c}\text { Mercado não é considerado quando do planejamento } \\
\text { dos custos. }\end{array}$ \\
\hline Redução dos custos com foco no cliente. & Redução de custos não voltada ao cliente. \\
\hline $\begin{array}{c}\text { Gerenciamento de custos efetuado por equipes } \\
\text { multifuncionais (de toda a empresa) }\end{array}$ & Custos monitorados apenas pelos contadores. \\
\hline $\begin{array}{c}\text { Envolve toda a cadeia de valor no planejamento } \\
\text { de custos }\end{array}$ & $\begin{array}{c}\text { Pouco ou nenhum envolvimento da cadeia de valor } \\
\text { no planejamento de custos. }\end{array}$ \\
\hline
\end{tabular}

Quadro 2: Custo-Meta e Custo Tradicional

Fonte: Elaborado pelo autor com base nos conceitos dos autores citados.

Ducati (2006) comenta que relata que apesar das suas críticas por valer-se de técnicas da engenharia de valor, o custeio-meta é muito utilizado por empresas japonesas e norte-americanas, principalmente as montadoras de veículos. A decisão de se adotar o custeio-meta tem como razão principal buscar um preço justo ao consumidor baseado no justo valor daquele produto, mas também, buscar reduzir o custo deste produto. 
Nesse sentido, a engenharia de valor, segundo Monden (1999, p. 98), representa a verdadeira essência do custeio-meta, pois, através dela busca-se mostrar as atividades que geram custos na etapa de projeto do produto e conhecer as atividades geradoras de custos é a base para sua redução.

A redução de valor na composição do preço do produto ou serviço envolve o processo de examinar cada componente de um produto para determinar se é possível ser reduzido, mantendo a funcionalidade e o desempenho do produto (ATKINSON et al., 2008).

Maher (2001) menciona que na engenharia de valor se avalia de forma sistemática todos os elementos relacionados com pesquisa e desenvolvimento, design dos produtos e processos, produção, marketing, distribuição e atenção aos clientes, visando a redução de custos e satisfação das necessidades dos clientes. Assim, a Engenharia de Valor busca a redução de custos eliminando ou reduzindo custos desnecessários no produto ou serviço. Cruz e Rocha (2008) consideram-na um conjunto de procedimentos que visa manter ou melhorar as funcionalidades de um produto, minimizando o custo total do consumidor.

Sakurai (1997) reforça que a engenharia de valor pode ser aplicada à produção Industrial e às prestadoras de serviços. As atividades de engenharia de valor podem ser divididas em três etapas:

a) Engenharia de abordagem zero: aplicada no planejamento do produto ou serviço;

b) Engenharia de valor da primeira abordagem: aplicada no estágio de desenho e desenvolvimento do produto ou serviço;

c) Engenharia de valor de segunda abordagem: aplicada no estágio de fabricação do produto, sendo possíveis somente melhorias incrementais no processo.

Segundo Cardoso e Beuren (2006), na primeira etapa encontram-se as ideias inovadoras, definindo a forma pela qual o produto ou serviço deve ser produzido ou executado. Na segunda etapa já ocorre a produção de produtos pilotos, onde se verifica se a estrutura existente é suficiente para a execução do projeto. Na última etapa ocorre 
a fabricação propriamente dita, onde as principais melhorias e as grandes reduções de custos já foram efetuadas.

\section{METODOLOGIA}

O objetivo do estudo é identificar os itens de importância na composição do custo- meta como ferramenta a ser considerada na formação do valor das mensalidades dos alunos que compõem a amostra da IES examinada, uma vez que a existência de outras possibilidades no mercado poderia acarretar na opção por outra instituição.

Utilizou-se uma amostragem por acessibilidade ou conveniência que, segundo Beuren (2004), normalmente é utilizada em pesquisas exploratórias ou qualitativas, nais quais existe uma menor cobrança no nível de precisão dos dados.

O estudo foi desenvolvido na Universidade Regional Integrada, Campus de Frederico Westphalen, Rio Grande do Sul, considerando três cursos de graduação (Letras, Ciências Contábeis e Farmácia) com valores de mensalidades distintas, contemplando um curso com valor de mensalidade menor, um com valor intermediário e um curso de maior valor.

A coleta de dados ocorreu por meio de aplicação de questionário enviado por email e também entregue pessoalmente aos acadêmicos. O questionário continha quatorze itens considerados como principais geradores de custos nas mensalidades dos alunos, aos quais deveriam ser atribuídas notas de 1 a 5 , conforme o grau de importância dado a cada um, considerando-se que os valores das mensalidades são atribuídos através do valor médio das demais instituições que desenvolvem este tipo de atividade. 
Utilização do custo-meta na formação de preços em entidades prestadoras de serviços educacionais. Alzenir José de Vargas, Jorge Eduardo Scarpin

Foram enviados 200 questionários para os alunos dos três cursos sendo que 191 retornaram respondidos, representando mais de $95 \%$ dos questionários enviados. Dos questionários respondidos, 176 foram usados na amostra, visto que 15 retornaram respondidos parcialmente. Os questionários respondidos e aproveitados representam mais de $92 \%$ dos questionários enviados.

Através da análise descritiva os dados foram examinados pela frequência, pela média ponderada e desvio-padrão das respostas obtidas, por meio da utilização de planilhas eletrônicas.

\section{ANÁLISE DOS RESULTADOS}

Os respondentes e respectivos cursos podem ser visualizados na Tabela 1.

Tabela 1: Composição dos respondentes

\begin{tabular}{|c|c|c|c|c|c|c|}
\hline Curso & Sem 2 & Sem 4 & Sem 6 & Sem 8 & Sem 10 & Total \\
\hline Ciências Contábeis & 0 & 29 & 41 & 28 & 0 & 98 \\
\hline Letras & 11 & 6 & 9 & 14 & 0 & 40 \\
\hline Farmácia & 13 & 0 & 11 & 11 & 3 & 38 \\
\hline
\end{tabular}

Fonte: Dados da pesquisa.

As diferenças identificadas na quantidade de alunos em cada curso estão relacionadas diretamente ao número total de alunos atualmente matriculados. A análise dos dados foi efetuada de forma individualizada, por curso.

Os itens seguintes foram examinados em dois blocos de respostas compostos por: EP - Extremamente importante e MI - Muito Importante, isto relacionado às atribuições importantes oferecidas no campus; e no bloco dois: PI - Pouco Importante e $\mathrm{NI}$ - Nada Importante. Os respondentes que assinalaram ID - Indiferentes, são considerados como respondentes neutros na análise. 
Utilização do custo-meta na formação de preços em entidades prestadoras de serviços

educacionais.

Alzenir José de Vargas, Jorge Eduardo Scarpin

Tabela 2: Atendimento disponibilizado pela secretaria do campus

\begin{tabular}{|c|c|c|c|c|c|c|c|c|c|c|}
\hline & \multicolumn{3}{|c|}{ EI } & \multicolumn{2}{|c|}{ MI } & \multicolumn{2}{|c|}{ ID } & \multicolumn{2}{|c|}{ PI } & \multicolumn{2}{c|}{ NI } \\
\hline Cursos & $\mathbf{F}$ & $\%$ & $\mathbf{F}$ & $\%$ & $\mathbf{F}$ & $\%$ & $\mathbf{F}$ & $\%$ & $\mathbf{F}$ & $\%$ \\
\hline Ciências Contábeis & 25 & 25,52 & 57 & 58,16 & 13 & 13,26 & 3 & 3,06 & 0 & 0,00 \\
\hline Letras & 27 & 67,50 & 10 & 25,00 & 2 & 5,00 & 1 & 2,50 & 0 & 0,00 \\
\hline Farmácia & 5 & 13,16 & 20 & 52,63 & 10 & 26,31 & 3 & 7,89 & 0 & 0,00 \\
\hline Geral & $\mathbf{5 7}$ & $\mathbf{3 2 , 3 9}$ & $\mathbf{8 7}$ & $\mathbf{4 9 , 4 3}$ & $\mathbf{2 5}$ & $\mathbf{1 4 , 2 0}$ & $\mathbf{7}$ & $\mathbf{3 , 9 8}$ & $\mathbf{0}$ & $\mathbf{0 , 0 0}$ \\
\hline
\end{tabular}

O atendimento disponibilizado pela secretaria do campus é considerado importante para mais de $81 \%$ dos respondentes, enquanto menos de $4 \%$ consideramno não importante, e, aproximadamente $14 \%$ mantêm-se indiferentes perante este serviço. Percebe-se, então, que o atendimento acadêmico desenvolvido pela secretaria é considerado importante para a maioria dos acadêmicos.

Tabela 3: Atendimento da tesouraria do campus

\begin{tabular}{|c|c|c|c|c|c|c|c|c|c|c|}
\hline & \multicolumn{3}{|c|}{ EI } & \multicolumn{2}{|c|}{ MI } & \multicolumn{2}{|c|}{ ID } & \multicolumn{2}{|c|}{ PI } & \multicolumn{2}{c|}{ NI } \\
\hline Cursos & F & $\%$ & F & \% & F & $\%$ & F & $\%$ & F & $\%$ \\
\hline Ciências Contábeis & 32 & 32,65 & 49 & 50,00 & 12 & 12,25 & 4 & 4,08 & 1 & 1,02 \\
\hline Letras & 31 & 77,50 & 6 & 15,00 & 3 & 7,50 & 0 & 0,00 & 0 & 0,00 \\
\hline Farmácia & 4 & 10,52 & 22 & 57,91 & 9 & 23,68 & 3 & 7,89 & 0 & 0,00 \\
\hline Geral & $\mathbf{6 7}$ & $\mathbf{3 8 , 0 7}$ & $\mathbf{7 7}$ & $\mathbf{4 3 , 7 5}$ & $\mathbf{2 4}$ & $\mathbf{1 3 , 6 3}$ & $\mathbf{7}$ & $\mathbf{3 , 9 8}$ & $\mathbf{1}$ & $\mathbf{0 , 5 7}$ \\
\hline
\end{tabular}

Os serviços de tesouraria são oferecidos aos acadêmicos para recebimento de mensalidades, taxas de serviços utilizados e também como centro de informações sobre questões econômicas e financeiras do campus. Esta função é considerada importante para mais de $81 \%$ dos respondentes, menos de $5 \%$ não consideram esta atividade importante. Aproximadamente $14 \%$ dos respondentes se posicionaram indiferentes e para menos de $5 \% 0$ atendimento da tesouraria não tem importância.

Tabela 4: Atendimento do Serviço de atendimento ao estudante - SAE

\begin{tabular}{|c|c|c|c|c|c|c|c|c|c|c|}
\hline & \multicolumn{2}{|c|}{ EI } & \multicolumn{2}{|c|}{ MI } & \multicolumn{2}{|c|}{ ID } & \multicolumn{2}{|c|}{ PI } & \multicolumn{2}{c|}{ NI } \\
\hline Cursos & $\mathbf{F}$ & $\%$ & $\mathbf{F}$ & $\%$ & $\mathbf{F}$ & $\%$ & $\mathbf{F}$ & $\%$ & $\mathbf{F}$ & $\%$ \\
\hline Ciências Contábeis & 41 & 41,84 & 33 & 33,67 & 16 & 16,33 & 5 & 5,10 & 3 & 3,06 \\
\hline Letras & 27 & 67,50 & 8 & 20,00 & 5 & 12,50 & 0 & 0,00 & 0 & 0,00 \\
\hline Farmácia & 11 & 28,95 & 18 & 47,37 & 8 & 21,05 & 1 & 2,63 & 0 & 0,00 \\
\hline Geral & $\mathbf{7 9}$ & $\mathbf{4 4 , 8 9}$ & $\mathbf{5 9}$ & $\mathbf{3 3 , 5 2}$ & $\mathbf{2 9}$ & $\mathbf{1 6 , 4 8}$ & $\mathbf{6}$ & $\mathbf{3 , 4 1}$ & $\mathbf{3}$ & $\mathbf{1 , 7 0}$ \\
\hline
\end{tabular}


Utilização do custo-meta na formação de preços em entidades prestadoras de serviços educacionais. Alzenir José de Vargas, Jorge Eduardo Scarpin

O atendimento ao estudante é um serviço que busca auxiliar os alunos na concessão de créditos educativos, orientação sobre moradias, transportes até o campus, bem como toda a informação que o acadêmico necessita a respeito da instituição. Mais de $78 \%$ dos acadêmicos consideram importante o serviço oferecido; apenas $5,11 \%$ classificam o serviço pouco ou nada importante. O destaque entre os cursos pesquisados é novamente para o curso de Letras com mais de $87 \%$ dos acadêmicos avaliando como importante o serviço oferecido.

Tabela 5: Atribuições da Coordenação do Curso

\begin{tabular}{|c|c|c|c|c|c|c|c|c|c|c|}
\hline & \multicolumn{3}{|c|}{ EI } & \multicolumn{2}{|c|}{ MI } & \multicolumn{2}{|c|}{ ID } & \multicolumn{2}{|c|}{ PI } & \multicolumn{1}{c|}{ NI } \\
\hline Cursos & $\mathbf{F}$ & $\%$ & $\mathbf{F}$ & $\%$ & $\mathbf{F}$ & $\%$ & $\mathbf{F}$ & $\%$ & $\mathbf{F}$ & $\%$ \\
\hline Ciências Contábeis & 40 & 40,82 & 43 & 43,88 & 11 & 11,22 & 2 & 2,04 & 2 & 2,04 \\
\hline Letras & 26 & 65,00 & 11 & 27,50 & 2 & 5,00 & 0 & 0,00 & 1 & 2,50 \\
\hline Farmácia & 20 & 52,63 & 14 & 36,84 & 2 & 5,26 & 2 & 5,26 & 0 & 0,00 \\
\hline Geral & $\mathbf{8 6}$ & $\mathbf{4 8 , 8 6}$ & $\mathbf{6 8}$ & $\mathbf{3 8 , 6 4}$ & $\mathbf{1 5}$ & $\mathbf{8 , 5 2}$ & $\mathbf{4}$ & $\mathbf{2 , 2 7}$ & $\mathbf{3}$ & $\mathbf{1 , 7 0}$ \\
\hline
\end{tabular}

Às coordenações de curso compete à gestão do curso, compreendendo a relação professor/aluno e toda a organização das atividades realizadas no curso. Esta função é considerada importante por mais de $87 \%$ dos acadêmicos e menos de $4 \%$ julgam pouco ou nada importante a função desempenhada pela coordenação.

Tabela 6: Atribuições dos professores

\begin{tabular}{|c|c|c|c|c|c|c|c|c|c|c|}
\hline & \multicolumn{2}{|c|}{ El } & \multicolumn{2}{|c|}{ MI } & \multicolumn{1}{|c|}{ ID } & \multicolumn{2}{|c|}{ PI } & \multicolumn{2}{|c|}{ NI } \\
\hline Cursos & $\mathbf{F}$ & $\%$ & $\mathbf{F}$ & $\%$ & $\mathbf{F}$ & $\%$ & $\mathbf{F}$ & $\%$ & $\mathbf{F}$ & $\%$ \\
\hline Ciências Contábeis & 70 & 71,43 & 24 & 24,49 & 4 & 4,08 & 0 & 0,00 & 0 & 0,00 \\
\hline Letras & 29 & 72,50 & 9 & 22,50 & 1 & 2,50 & 1 & 2,50 & 0 & 0,00 \\
\hline Farmácia & 19 & 50,00 & 15 & 39,47 & 1 & 2,63 & 1 & 2,63 & 2 & 5,26 \\
\hline Geral & $\mathbf{1 1 8}$ & $\mathbf{6 7 , 0 5}$ & $\mathbf{4 8}$ & $\mathbf{2 7 , 2 7}$ & $\mathbf{6}$ & $\mathbf{3 , 4 1}$ & $\mathbf{2}$ & $\mathbf{1 , 1 4}$ & $\mathbf{2}$ & $\mathbf{1 , 1 4}$ \\
\hline
\end{tabular}

A modalidade de ensino da instituição é presencial, contando com professores em atividades integrais na sala de aula, com contato diário com os acadêmicos. Mais de $92 \%$ confirmam a importância das atribuições conferidas aos professores. Menos de $3 \%$ consideram pouco ou nada importante as atribuições dos professores na sua formação. As atribuições conferidas aos professores se justificam pelo nível de qualificação dos 
Utilização do custo-meta na formação de preços em entidades prestadoras de serviços educacionais. Alzenir José de Vargas, Jorge Eduardo Scarpin

mesmos, lembrando que mais de $75 \%$ possuem formação stricto sensu em nível de mestrado e doutorado.

Tabela 7: Estrutura da biblioteca

\begin{tabular}{|c|c|c|c|c|c|c|c|c|c|c|}
\hline & \multicolumn{2}{|c|}{ El } & \multicolumn{2}{|c|}{ MI } & \multicolumn{2}{|c|}{ ID } & \multicolumn{3}{|c|}{ PI } & \multicolumn{2}{c|}{ NI } \\
\hline Cursos & $\mathbf{F}$ & $\%$ & $\mathbf{F}$ & $\%$ & $\mathbf{F}$ & $\%$ & $\mathbf{F}$ & $\%$ & $\mathbf{F}$ & $\%$ \\
\hline Ciências Contábeis & 66 & 67,35 & 32 & 32,65 & 0 & 0,00 & 0 & 0,00 & 0 & 0,00 \\
\hline Letras & 28 & 70,00 & 9 & 22,50 & 2 & 5,00 & 1 & 2,50 & 0 & 0,00 \\
\hline Farmácia & 27 & 71,05 & 9 & 23,68 & 1 & 2,63 & 0 & 0,00 & 1 & 2,63 \\
\hline Geral & $\mathbf{1 2 1}$ & $\mathbf{6 8 , 7 5}$ & $\mathbf{5 0}$ & $\mathbf{2 8 , 4 1}$ & $\mathbf{3}$ & $\mathbf{1 , 7 0}$ & $\mathbf{1}$ & $\mathbf{0 , 5 7}$ & $\mathbf{1}$ & $\mathbf{0 , 5 7}$ \\
\hline
\end{tabular}

A biblioteca da instituição possui uma estrutura moderna e obras atualizadas, havendo atualmente mais de 70.000 exemplares à disposição dos acadêmicos. Confirma-se a boa estrutura e a importância que a biblioteca tem na percepção dos acadêmicos quando mais de $97 \%$ concordam ser importante a estrutura oferecida pela instituição; com um percentual de pouco mais de $1 \%$ alegando ser ela pouco ou nada importante. Os investimentos realizados constantemente procuram garantir a utilização dos acervos pelos acadêmicos.

Tabela 8: Estrutura das salas de aula

\begin{tabular}{|c|c|c|c|c|c|c|c|c|c|c|}
\hline & \multicolumn{2}{|c|}{ El } & \multicolumn{2}{|c|}{ MI } & \multicolumn{2}{|c|}{ ID } & \multicolumn{2}{|c|}{ PI } & \multicolumn{2}{c|}{ NI } \\
\hline Cursos & $\mathbf{F}$ & $\%$ & $\mathbf{F}$ & $\%$ & $\mathbf{F}$ & $\%$ & $\mathbf{F}$ & $\%$ & $\mathbf{F}$ & $\%$ \\
\hline Ciências Contábeis & 48 & 48,98 & 43 & 43,88 & 4 & 4,08 & 3 & 3,06 & 0 & 0,00 \\
\hline Letras & 21 & 52,50 & 12 & 30,00 & 5 & 12,50 & 1 & 2,50 & 1 & 2,50 \\
\hline Farmácia & 15 & 39,47 & 14 & 36,84 & 9 & 23,68 & 0 & 0,00 & 0 & 0,00 \\
\hline Geral & $\mathbf{8 4}$ & $\mathbf{4 7 , 7 3}$ & $\mathbf{6 9}$ & $\mathbf{3 9 , 2 0}$ & $\mathbf{1 8}$ & $\mathbf{1 0 , 2 3}$ & $\mathbf{4}$ & $\mathbf{2 , 2 7}$ & $\mathbf{1}$ & $\mathbf{0 , 5 7}$ \\
\hline
\end{tabular}

As condições oferecidas aos acadêmicos procuram atender às suas expectativas. A instituição realiza investimentos a fim de oferecer uma acomodação adequada. Na opinião de mais de $86 \%$ dos respondentes é importante a estrutura oferecida em termos de salas de aula. Mais de $92 \%$ dos acadêmicos do curso de Ciências Contábeis assinalam a importância da estrutura oferecida, cujas salas estão equipadas com assentos estofados oferecendo um maior conforto aos alunos. 
Utilização do custo-meta na formação de preços em entidades prestadoras de serviços

educacionais.

Alzenir José de Vargas, Jorge Eduardo Scarpin

Tabela 9: Estrutura e acessibilidade nos estacionamentos

\begin{tabular}{|c|c|c|c|c|c|c|c|c|c|c|}
\hline & \multicolumn{2}{|c|}{ EI } & \multicolumn{2}{|c|}{ MI } & \multicolumn{2}{|c|}{ ID } & \multicolumn{2}{|c|}{ PI } & \multicolumn{2}{c|}{ NI } \\
\hline Cursos & $\mathbf{F}$ & $\%$ & $\mathbf{F}$ & $\%$ & $\mathbf{F}$ & $\%$ & $\mathbf{F}$ & $\%$ & $\mathbf{F}$ & $\%$ \\
\hline Ciências Contábeis & 31 & 31,63 & 38 & 38,78 & 18 & 18,37 & 8 & 8,16 & 3 & 3,06 \\
\hline Letras & 16 & 40,00 & 13 & 32,50 & 10 & 25,00 & 0 & 0,00 & 1 & 2,50 \\
\hline Farmácia & 5 & 13,16 & 15 & 39,47 & 13 & 34,21 & 5 & 13,16 & 0 & 0,00 \\
\hline Geral & $\mathbf{5 2}$ & $\mathbf{2 9 , 5 5}$ & $\mathbf{6 6}$ & $\mathbf{3 7 , 5 0}$ & $\mathbf{4 1}$ & $\mathbf{2 3 , 3 0}$ & $\mathbf{1 3}$ & $\mathbf{7 , 3 9}$ & $\mathbf{4}$ & $\mathbf{2 , 2 7}$ \\
\hline
\end{tabular}

O serviço de estacionamento é oferecido de forma gratuita aos acadêmicos, porém muitos alunos utilizam o transporte coletivo para o acesso à instituição. Isso se evidencia pelo fato dos acadêmicos se mostrarem indiferentes ou responderem como pouco importante a questão relacionada ao serviço de estacionamento. Mesmo assim, mais de $67 \%$ dizem ser importante a estrutura oferecida nos estacionamentos com um custo atribuído indiretamente ao aluno.

Tabela 10: Estrutura e acessibilidade nos laboratórios

\begin{tabular}{|c|c|c|c|c|c|c|c|c|c|c|}
\hline & \multicolumn{3}{|c|}{ EI } & \multicolumn{2}{|c|}{ MI } & \multicolumn{2}{|c|}{ ID } & \multicolumn{2}{|c|}{ PI } & \multicolumn{2}{c|}{ NI } \\
\hline Cursos & $\mathbf{F}$ & $\%$ & $\mathbf{F}$ & $\%$ & $\mathbf{F}$ & $\%$ & $\mathbf{F}$ & $\%$ & $\mathbf{F}$ & $\%$ \\
\hline Ciências Contábeis & 43 & 43,88 & 36 & 36,73 & 12 & 12,24 & 2 & 2,04 & 5 & 5,10 \\
\hline Letras & 21 & 52,50 & 14 & 35,00 & 2 & 5,00 & 2 & 5,00 & 1 & 2,50 \\
\hline Farmácia & 32 & 84,21 & 5 & 13,16 & 1 & 2,63 & 0 & 0,00 & 0 & 0,00 \\
\hline Geral & $\mathbf{9 6}$ & $\mathbf{5 4 , 5 5}$ & $\mathbf{5 5}$ & $\mathbf{3 1 , 2 5}$ & $\mathbf{1 5}$ & $\mathbf{8 , 5 2}$ & $\mathbf{4}$ & $\mathbf{2 , 2 7}$ & $\mathbf{6}$ & $\mathbf{3 , 4 1}$ \\
\hline
\end{tabular}

Todos os cursos oferecem uma estrutura de laboratórios específicos em cada área. Sobressai-se o curso de Farmácia com mais de 97\% considerando importante a estrutura oferecida nos seus laboratórios. A média entre os pesquisados fica aproximadamente em $86 \%$. Dentre a estrutura de custos que compõem o valor da mensalidade, os dispêndios financeiros aplicados nos laboratórios representam um valor significativo. 
Utilização do custo-meta na formação de preços em entidades prestadoras de serviços

educacionais.

Alzenir José de Vargas, Jorge Eduardo Scarpin

Tabela 11: Serviços do restaurante universitário

\begin{tabular}{|c|c|c|c|c|c|c|c|c|c|c|}
\hline & \multicolumn{2}{|c|}{ El } & \multicolumn{2}{|c|}{ MI } & \multicolumn{2}{|c|}{ ID } & \multicolumn{2}{|c|}{ PI } & \multicolumn{2}{c|}{ NI } \\
\hline Cursos & $\mathbf{F}$ & $\%$ & $\mathbf{F}$ & $\%$ & $\mathbf{F}$ & $\%$ & $\mathbf{F}$ & $\%$ & $\mathbf{F}$ & $\%$ \\
\hline Ciências Contábeis & 5 & 5,10 & 31 & 31,63 & 33 & 33,67 & 18 & 18,37 & 11 & 11,22 \\
\hline Letras & 12 & 30,00 & 12 & 30,00 & 10 & 25,00 & 6 & 15,00 & 0 & 0,00 \\
\hline Farmácia & 9 & 23,68 & 8 & 21,05 & 13 & 34,21 & 3 & 7,89 & 5 & 13,16 \\
\hline Geral & $\mathbf{2 6}$ & $\mathbf{1 4 , 7 7}$ & $\mathbf{5 1}$ & $\mathbf{2 8 , 9 8}$ & $\mathbf{5 6}$ & $\mathbf{3 1 , 8 2}$ & $\mathbf{2 7}$ & $\mathbf{1 5 , 3 4}$ & $\mathbf{1 6}$ & $\mathbf{9 , 0 9}$ \\
\hline
\end{tabular}

O restaurante universitário é um serviço terceirizado, que tem o propósito de oferecer alimentação e bebidas. Menos de $44 \%$ dos acadêmicos acreditam que os serviços oferecidos são importantes. A maioria se posiciona indiferentemente ou atribuindo pouca importância à este serviço. Entre os acadêmicos do curso de Ciências Contábeis, menos de $37 \%$ consideram importante o serviço. A estrutura de bares fora do campus é um indicativo da pouca importância apresentada pelos acadêmicos.

Tabela 12: Serviços e produtos da livraria

\begin{tabular}{|c|c|c|c|c|c|c|c|c|c|c|}
\hline & \multicolumn{3}{|c|}{ EI } & \multicolumn{2}{|c|}{ MI } & \multicolumn{2}{|c|}{ ID } & \multicolumn{2}{|c|}{ PI } & \multicolumn{2}{c|}{ NI } \\
\hline Cursos & $\mathbf{F}$ & $\%$ & $\mathbf{F}$ & $\%$ & $\mathbf{F}$ & $\%$ & $\mathbf{F}$ & $\%$ & $\mathbf{F}$ & $\%$ \\
\hline Ciências Contábeis & $\mathbf{2 8}$ & $\mathbf{2 8 , 5 7}$ & 40 & 40,82 & 20 & 20,41 & 6 & 6,12 & 4 & 4,08 \\
\hline Letras & 16 & 40,00 & 15 & 37,50 & 3 & 7,50 & 6 & 15,00 & 0 & 0,00 \\
\hline Farmácia & 11 & 28,95 & 21 & 55,26 & 5 & 13,16 & 0 & 0,00 & 1 & 2,63 \\
\hline Geral & $\mathbf{5 5}$ & $\mathbf{3 1 , 2 5}$ & $\mathbf{7 6}$ & $\mathbf{4 3 , 1 8}$ & $\mathbf{2 8}$ & $\mathbf{1 5 , 9 1}$ & $\mathbf{1 2}$ & $\mathbf{6 , 8 2}$ & $\mathbf{5}$ & $\mathbf{2 , 8 4}$ \\
\hline
\end{tabular}

Os serviços oferecidos pela livraria compreendem a comercialização de livros, materiais escolares e fotocópias. A livraria está localizada em dois locais internos no campus, configurando-se, também, em um serviço terceirizado, sem ingerência da instituição, cuja estrutura física é disponibilizada para o serviço descrito. Mais de $74 \%$ dos acadêmicos asseguram que as funções desempenhadas pela livraria são importantes. 
Utilização do custo-meta na formação de preços em entidades prestadoras de serviços

educacionais.

Alzenir José de Vargas, Jorge Eduardo Scarpin

Tabela 13: Acesso ao portal Acadêmico - URINET

\begin{tabular}{|c|c|c|c|c|c|c|c|c|c|c|}
\hline & \multicolumn{2}{|c|}{ EI } & \multicolumn{2}{|c|}{ MI } & \multicolumn{2}{|c|}{ ID } & \multicolumn{2}{|c|}{ PI } & \multicolumn{2}{c|}{ NI } \\
\hline Cursos & $\mathbf{F}$ & $\%$ & $\mathbf{F}$ & $\%$ & $\mathbf{F}$ & $\%$ & $\mathbf{F}$ & $\%$ & $\mathbf{F}$ & $\%$ \\
\hline Ciências Contábeis & 59 & 60,20 & 34 & 34,69 & 5 & 5,10 & 0 & 0,00 & 0 & 0,00 \\
\hline Letras & 28 & 70,00 & 7 & 17,50 & 4 & 10,00 & 1 & 2,50 & 0 & 0,00 \\
\hline Farmácia & 22 & 57,89 & 11 & 28,95 & 4 & 10,53 & 0 & 0,00 & 1 & 2,63 \\
\hline Geral & $\mathbf{1 0 9}$ & $\mathbf{6 1 , 9 3}$ & $\mathbf{5 2}$ & $\mathbf{2 9 , 5 5}$ & $\mathbf{1 3}$ & $\mathbf{7 , 3 9}$ & $\mathbf{1}$ & $\mathbf{0 , 5 7}$ & $\mathbf{1}$ & $\mathbf{0 , 5 7}$ \\
\hline
\end{tabular}

Os serviços acadêmicos e financeiros são oferecidos online a todos os acadêmicos permitindo acessar resultados de avaliações, presenças em aula, currículo do curso, receber e enviar e-mail e mensagem, acessar material disponibilizado pelo professor, realizar rematrícula, bem como verificar toda a situação financeira junto à instituição. O sistema disponibilizado aos acadêmicos é desenvolvido e mantido por uma equipe de profissionais de $\mathrm{TI}$ da própria instituição, representando custos significativos à instituição. Constata-se que mais de $91 \%$, em média, dos acadêmicos consideram importante este serviço oferecido, sendo que $94 \%$ dos respondentes representam o curso de Ciências Contábeis.

Tabela 14: Serviços de comunicação entre a Instituição e o Aluno

\begin{tabular}{|c|c|c|c|c|c|c|c|c|c|c|}
\hline & \multicolumn{3}{|c|}{ EI } & \multicolumn{2}{|c|}{ MI } & \multicolumn{2}{|c|}{ ID } & \multicolumn{2}{|c|}{ PI } & \multicolumn{2}{c|}{ NI } \\
\hline Cursos & $\mathbf{F}$ & $\%$ & $\mathbf{F}$ & $\%$ & $\mathbf{F}$ & $\%$ & $\mathbf{F}$ & $\%$ & $\mathbf{F}$ & $\%$ \\
\hline Ciências Contábeis & 43 & 43,88 & 41 & 41,84 & 11 & 11,22 & 3 & 3,06 & 0 & 0,00 \\
\hline Letras & 26 & 65,00 & 13 & 32,50 & 1 & 2,50 & 0 & 0,00 & 0 & 0,00 \\
\hline Farmácia & 14 & 36,84 & 19 & 50,00 & 4 & 10,53 & 1 & 2,63 & 0 & 0,00 \\
\hline Geral & $\mathbf{8 3}$ & $\mathbf{4 7 , 1 6}$ & $\mathbf{7 3}$ & $\mathbf{4 1 , 4 8}$ & $\mathbf{1 6}$ & $\mathbf{9 , 0 9}$ & $\mathbf{4}$ & $\mathbf{2 , 2 7}$ & $\mathbf{0}$ & $\mathbf{0 , 0 0}$ \\
\hline
\end{tabular}

A instituição garante linha telefônica com acesso gratuito a fim de facilitar o contato com os acadêmicos, além de uma diversidade de e-mails para todos os setores do campus e uma ouvidoria que está disponível a receber informações, reclamações e elogios, sendo os custos mantidos pelo campus. Mais de $88 \%$ dos acadêmicos entendem ser importante a comunicação com o campus. Destaca-se, neste item, o curso de Letras com mais de $97 \%$ considerando importante estes serviços. 
Utilização do custo-meta na formação de preços em entidades prestadoras de serviços

educacionais.

Alzenir José de Vargas, Jorge Eduardo Scarpin

Tabela 15: Realização de atividades educacionais extraclasse

\begin{tabular}{|c|c|c|c|c|c|c|c|c|c|c|}
\hline & \multicolumn{2}{|c|}{ EI } & \multicolumn{2}{|c|}{ MI } & \multicolumn{2}{|c|}{ ID } & \multicolumn{2}{|c|}{ PI } & \multicolumn{2}{c|}{ NI } \\
\hline Cursos & F & $\%$ & F & $\%$ & F & $\%$ & F & $\%$ & F & $\%$ \\
\hline Ciências Contábeis & 65 & 66,33 & 25 & 25,51 & 5 & 5,10 & 1 & 1,02 & 2 & 2,04 \\
\hline Letras & 31 & 77,50 & 8 & 20,00 & 0 & 0,00 & 1 & 2,50 & 0 & 0,00 \\
\hline Farmácia & 27 & 71,05 & 9 & 23,68 & 2 & 5,26 & 0 & 0,00 & 0 & 0,00 \\
\hline Geral & $\mathbf{1 2 3}$ & $\mathbf{6 9 , 8 9}$ & $\mathbf{4 2}$ & $\mathbf{2 3 , 8 6}$ & $\mathbf{7}$ & $\mathbf{3 , 9 8}$ & $\mathbf{2}$ & $\mathbf{1 , 1 4}$ & $\mathbf{2}$ & $\mathbf{1 , 1 4}$ \\
\hline
\end{tabular}

A realização de atividades acadêmicas fora de sala de aula tem o objetivo de complementar os conhecimentos adquiridos e, ainda, propiciar o contato com outras atividades e profissionais especializados em assuntos específicos discutidos. A instituição disponibiliza infraestrutura de salas, auditórios e equipamentos para a realização destas atividades no próprio campus. Mais de 93\%, em média, dos alunos dos cursos valorizam estas atividades, com destaque para o curso de Letras com mais de $97 \%$ dos acadêmicos considerando esta atividade importante.

\section{CONSIDERAÇÕES FINAIS}

O presente estudo traz uma contribuição aos demais estudos apresentados em relação aos aspectos considerados na composição do valor das mensalidades de cursos de graduação em uma instituição de ensino superior privado. Foram avaliados os principais itens de custos que impactam no valor das mensalidades cobradas.

No aspecto do atendimento, verifica-se a importância que os acadêmicos dão independente do setor da instituição. $O$ atendimento é efetuado por pessoal treinado e qualificado em cada um dos setores da instituição, constituindo-se um dos aspectos mais representativos na composição dos custos que compõem o valor da mensalidade. Devido à representatividade dessa constatação, cabe à instituição investir em treinamento e agilizar o atendimento, gerando uma satisfação maior aos acadêmicos e, consequentemente, permitindo uma redução no custo operacional.

A instituição oferece ensino presencial. Dessa forma, o custo direto relacionado com coordenação de cursos e com professores representa parte significativa no valor 
cobrado nas mensalidades. Os acadêmicos também têm a percepção da importância destes fatores de custos no valor pago para a instituição, conforme resultados apresentados nas tabelas cinco e seis.

Com a finalidade de atender à demanda de aproximadamente 3.000 alunos em dezoito cursos de graduação, mais de vinte cursos de especialização e escola de ensino básico, a instituição oferece condições de salas de aula, bibliotecas, estacionamentos e laboratórios capazes de atender satisfatoriamente aos acadêmicos. Os gastos com manutenção e reposição representam um custo significativo a ser repassado para o acadêmico, no entanto, há o reconhecimento da importância desta estrutura pelos acadêmicos, principalmente no que diz respeito à biblioteca e aos laboratórios de pesquisa.

Nos serviços auxiliares de restaurante e livraria, a instituição não possui ingerência nestes segmentos, mas estes estão alocados em prédios da instituição, o que inclui custos relacionados à manutenção e seguro dos mesmos, visando oferecer condições diferenciadas aos acadêmicos. Contudo, na opinião da maioria dos acadêmicos os serviços do restaurante universitário são indiferentes ou pouco significativos. Tal aspecto deve ser considerado nos custos gerados neste setor, se realmente se justifica o valor repassado na mensalidade.

As formas de comunicação oferecidas pela instituição, tanto online quanto via telefone, representam um fator de custos considerável, uma vez que o desenvolvimento e manutenção do sistema online são próprios, bem como a manutenção de linha telefônica sem custo direto para o acadêmico, resultando em alocação nos valores das mensalidades. Oferecer um canal de comunicação direta com os acadêmicos é uma necessidade e representa um contato permanente e hábito rotineiro destes estarem informados do que acontece na instituição.

A oferta de cursos na modalidade presencial possibilita que a instituição ofereça cursos, palestras e seminários complementares às atividades de sala de aula, precisando da disponibilização de estrutura suficiente para atender esta demanda, 
porém, por meio da importância destacada pelos acadêmicos, se justifica a existência destes fatores de custos.

Por fim, pode se destacar que a utilização do custo-meta na definição dos fatores que compõem o preço de um produto ou serviço pode ser definida pela importância que o cliente demonstra na sua aquisição. Com base na amostra pesquisada, é possível identificar em cada item examinado a importância que o acadêmico atribui ao valor que ele está pagando, da mesma forma serve para a instituição avaliar o que realmente é importante para o seu cliente.

\section{REFERÊNCIAS}

ANSARI, Shahid et al. (1997). Target Costing: the Next Frontier in Strategic Cost Management. New York: McGraw-Hill, 1997.

ATKINSON, Anthony; BANKER Rajiv D.; KAPLAN, Robert S.; YOUNG S. Mark. (2008). Contabilidade Gerencial. (2 ed.). São Paulo: Atlas.

BEUREN, Ilse Maria. et al. (2009). Como elaborar trabalhos monográficos em contabilidade. (3 ed.). São Paulo: Atlas.

CARDOSO, Rodrigo dos Santos; BEUREN, Ilse Maria. (2006). O custo meta no desenvolvimento de novos produtos: um estudo de caso em Indústria de Conserva de Pescado. Revista Brasileira de Gestão de Negócios. São Paulo, v. 8, n. 22, p. 13-22.

CHIACENATO, Idalberto. (1990). Iniciação ao planejamento e controle da produção. São Paulo: McGraw-Hill.

CHIMENDES, Vanessa Cristhina Gatto; ZACCARO, Christiano Henrique. (2008). Proposta de um modelo de aplicação de custo-alvo no setor de serviços. Revista de Administração da Fatea. São Paulo, v. 1, n. 1, p. 1-11.

CRUZ, Cassia Vanessa Olak Alves; ROCHA Wellington. (2008). Custo-alvo: reflexões sobre definições, finalidades e procedimentos. In: CONGRESSO BRASILEIRO DE CUSTOS, 15, Curitiba. Anais...São Paulo.

DUCATI, Erves. (2006). O custo-meta e o custeio variável: é possível essa integração? In: ENANPAD, 30, Salvador. Anais...Salvador. 
HORNGREN, Charles T.; FOSTER, George; DATAR, Srikant M. (1997). Contabilidade de custos. Rio de Janeiro: LTC.

KNUTH, Valdecir. (2005). Aplicação do sistema de custeio meta às empresas do setor faccionista de costura: um estudo de caso. Santa Catarina, 2005. 165f. (Dissertação de Mestrado). Centro de Ciências Sociais Aplicadas, Universidade Regional de Blumenau. Blumenau.

MARTINS, Eliseu. (2006). Contabilidade de custos. (9 ed.). São Paulo: Atlas.

MONDEN, Yasuhiro. (1999). Sistemas de redução de custos: custo-alvo e custo kaizen. Porto Alegre: Bookman.

ROCHA, Wellington. (1999). Contribuição ao estudo de um modelo conceitual de sistema de informações da gestão estratégica. 148p. (Tese de Doutorado). Faculdade de Economia, Administração e Contabilidade da Universidade de São Paulo. São Paulo.

SAKURAI, Michiharu. (1997). Gerenciamento integrado de custos. São Paulo: Atlas.

SCARPIN Jorge Eduardo; ROCHA, Wellington. (2000). Target Costing: abordagem conceitual e histórica. In: CONGRESSO BRASILEIRO DE CUSTOS, 7, 2000, Recife. Anais...São Paulo. 1 CD.

WERNKE Rodney. (2008). Gestão de custos. (2 ed.). São Paulo: Atlas.

Data de Submissão: 05/07/2012

Data de Aceite: 12/03/2014 